\title{
Shifts of Effective Connectivity within a Language Network during Rhyming and Spelling
}

\author{
Tali Bitan, ${ }^{1}$ James R. Booth, ${ }^{1}$ Janet Choy, ${ }^{1}$ Douglas D. Burman, ${ }^{1}$ Darren R. Gitelman, ${ }^{2}$ and M.-Marsel Mesulam ${ }^{2}$ \\ ${ }^{1}$ Northwestern University, Evanston, Illinois 60208, and ${ }^{2}$ Northwestern University Medical School, Chicago, Illinois 60611
}

\begin{abstract}
We used functional magnetic resonance imaging to examine task-specific modulations of effective connectivity within a left-hemisphere language network during spelling and rhyming judgments on visually presented words. We identified sites showing task-specific activations for rhyming in the lateral temporal cortex (LTC) and for spelling in the intraparietal sulcus (IPS). The inferior frontal gyrus (IFG) and fusiform gyrus were engaged by both tasks. Dynamic causal modeling showed that each task preferentially strengthened modulatory influences converging on its task-specific site (LTC for rhyming, IPS for spelling). These remarkably selective and symmetrical findings demonstrate that the nature of the behavioral task dynamically shifts the locus of integration (or convergence) to the network component specialized for that task. Furthermore, they suggest that the role of the task-selective areas is to provide a differential synthesis of incoming information rather than providing differential control signals influencing the activity of other network components. Our findings also showed that switching tasks led to changes in the target area influenced by the IFG, suggesting that the IFG may play a pivotal role in setting the cognitive context for each task. We propose that task-dependent shifts in effective connectivity are likely to be mediated through top-down modulations from the IFG to the task-selective regions in a way that differentially enhances their sensitivity to incoming word-form information.
\end{abstract}

Key words: convergence zones; fMRI; inferior frontal gyrus; fusiform gyrus; dynamic causal modeling; reading

\section{Introduction}

Functional neuroimaging studies aim to identify network components that are selectively engaged by cognitive tasks. However, a network could shift from one behavioral goal to another not because of differences in the distribution of activations, but because of differences in the interactions among its components (Mesulam, 1981, 1998; Damasio, 1989; McIntosh, 2000). Analyses of effective connectivity (the influence that one brain region exerts on another) and its nondirectional counterpart known as functional connectivity have, in fact, shown that network components can display task-dependent alterations in their interactions (McIntosh et al., 1994; Horwitz et al., 1998; Pugh et al., 2000; Chaminade and Fonlupt, 2003; Homae et al., 2003). Components of distributed networks serve multiple roles including the integration of convergent inputs, the binding of distributed information, the relay of information from one region to another, and the control of neural activity within other network components (Mesulam, 1998). The goal of the current study is to identify the ways in which two different lexical processing tasks alter the dynamic interactions within a left-hemisphere language network. Specifically, we wanted to explore whether task-specific shifts in the direction of sensory-fugal processing and in the lo-

Received Jan. 26, 2005; revised April 19, 2005; accepted April 23, 2005.

This work was supported by grants from the National Institute of Child Health and Human Development (HD042049), the National Institute of Deafness and Other Communication Disorders (DC06149), and the National Institute on Aging [P50 AG06882 and P30 AG097761 (M.-M.M.); K23 AG00940 (D.R.G.)].

Correspondence should be addressed to Tali Bitan, Department of Communication Sciences and Disorders, Frances Searle Building, 2250 North Campus Drive, Evanston, IL 60208. E-mail: t-bitan@northwestern.edu. D01:10.1523/JNEUROSCI.0864-05.2005

Copyright $\odot 2005$ Society for Neuroscience $\quad$ 0270-6474/05/255397-07\$15.00/0 cation of convergence could be identified, as conceptualized by the models of Damasio (1989) and Mesulam (1981, 1998).

Two studies that examined effective connectivity among brain regions in language tasks (Bullmore et al., 2000; Mechelli et al., 2002) included the left fusiform gyrus (FG) as the sensory input source and the left inferior frontal region and superior temporal gyrus as additional network components. However, neither study was designed to identify the alterations of effective connectivity within network components that were selectively engaged by specific cognitive operations.

Dynamic causal modeling (DCM) is a nonlinear systems identification procedure that uses Bayesian estimation to make inferences about effective connectivity between neural systems and how it is affected by experimental conditions. Because connectivity in DCM is measured through the coupling of changes in imaging signals, rather than anatomically, a significant unidirectional modulatory influence of one brain region on another does not necessarily reflect the presence of a direct and unidirectional anatomical connection. Instead, the connectivity revealed by DCM reflects the inferred direction of neural influences that are specific to the imaging conditions and that may be mediated through interneurons or other brain regions not explicitly included in the model.

In the current study, we examined effective connectivity within a distributed language network while subjects performed spelling or rhyming judgments on visually presented words. Four left hemisphere regions were included in the network: the FG, the inferior frontal gyrus (IFG), the cortex of the intraparietal sulcus (IPS), and the middle portion of lateral temporal cortex (LTC). We examined task-specific shifts in the effective connectivity us- 
ing the DCM approach (Friston et al., 2003) on a subset of the data from Booth et al. (2002). We found that changes in cognitive goals caused major shifts in the site of convergence, in the routing of sensory information, and in the targeting of topdown control.

\section{Materials and Methods}

Subjects. Fourteen adults, five males and nine females, 20-35 years of age, (mean age, 25 years), participated in this study. All partici-

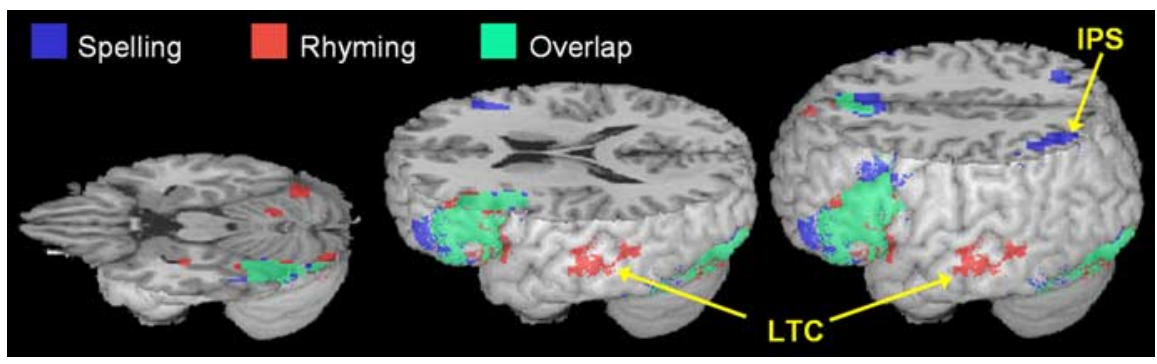

Figure 1. Group activation for the spelling and rhyming tasks compared with baseline. Extent threshold, $>50$ voxels. pants were right-handed, native Englishspeaking university students with no diagnosed neurological/psychiatric disorders or language/reading disabilities.

Stimuli and tasks. The stimuli and tasks were described in detail previously (Booth et al., 2002). In both the spelling and rhyming tasks, three words were presented sequentially, and the participant had to determine whether the final word matched either of the two previous words according to a predefined rule. In the spelling task, participants determined whether the final word had the same rhyme spelling as either of the first two words. The rhyme included all letters after the first consonant or consonant cluster (Bowey, 1990). In the rhyming task, participants determined whether the final word rhymed with either of the first two words. Participants indicated their judgment by pressing one of two buttons. For both the spelling and rhyming tasks, one-half of the target trials contained a target word that rhymed and was orthographically similar to one of the preceding two words [i.e., had the same rhyme (like hold-cold)]. The other half contained a target word that rhymed but was orthographically dissimilar to one of the preceding two words (e.g., hope-soap). In addition, one-half of the correct trials involved a match to the first stimulus, and one-half involved a match to the second stimulus. Sixty percent of the trials involved a match, and $40 \%$ involved a nonmatch. In the perceptual control task, the experimental setup and timing for the control blocks was exactly the same as for the word blocks, except the three stimuli were abstract, nonlinguistic symbols consisting of straight lines (e.g., //). The subjects were asked to determine whether the final line matched either of the first two lines.

Experimental procedure. The spelling and the rhyming tasks were each administered in a separate 9 min run, which was preceded by written instructions to the subject. Each task procedure consisted of 10 blocks of $54 \mathrm{~s}$, in which five experimental blocks of either the spelling or the rhyming task alternated with five control blocks. In each trial for the experimental blocks, three consecutive words were presented, each word for $800 \mathrm{~ms}$ followed by a $200 \mathrm{~ms}$ blank interval. Participants had $2000 \mathrm{~ms}$ to respond. Each trial lasted a total of $5000 \mathrm{~ms}$. Each block began with a $4 \mathrm{~s}$ instruction followed by 10 trials.

Functional magnetic resonance imaging data acquisition. Images were acquired using a 1.5 tesla General Electric (Milwaukee, WI) scanner with echo planar imaging method. The scanning parameters were the following: repetition time (TR), $3000 \mathrm{~ms}$; echo time (TE), $40 \mathrm{~ms}$; flip angle, $90^{\circ}$; matrix size, $64 \times 64$; field of view, $22 \mathrm{~cm}$; slice thickness, $4 \mathrm{~mm}$; number of slices, 32 . These scanning parameters resulted in a $3.437 \times 3.437 \times 4$ $\mathrm{mm}$ voxel size. Two runs of $9 \mathrm{~min}$ (180 images) were acquired, one with each experimental task. A high-resolution, T1-weighted threedimensional image was also acquired (spoiled gradient-recalled acquisition in a steady state; TR, $21 \mathrm{~ms}$; TE, $8 \mathrm{~ms}$; flip angle, $20^{\circ}$; matrix size, $256 \times 256$; field of view, $22 \mathrm{~cm}$; slice thickness, $1 \mathrm{~mm}$ ).

Image data analysis. Data analysis was performed using statistical parametric mapping (SPM2) (http://www.fil.ion.ucl.ac.uk/spm). The images were spatially realigned to the first volume to correct for head movements. No individual runs had $>2.5 \mathrm{~mm}$ maximum displacement. Sinc interpolation was used to minimize timing errors between slices (Henson et al., 1999). The functional images were coregistered with the anatomical image and normalized to the standard T1 template volume (Montreal Neurological Institute, Montreal, Quebec, Canada). The data were then smoothed with a $7 \mathrm{~mm}$ isotropic Gaussian kernel.

Statistical analyses at the first level were calculated using an epoch-

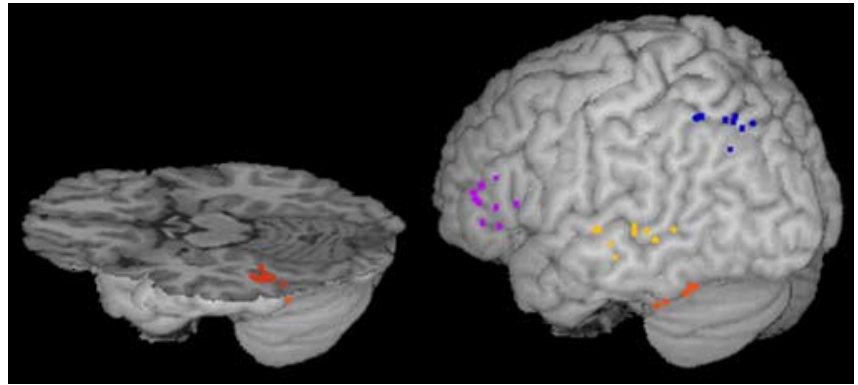

Figure 2. Center of ROIs in individual subjects. Pink, IFG; orange, FG; yellow, LTC; blue, IPS.

based design, with the two tasks (spelling and rhyming) as the conditions of interest. Images from the two separate runs were entered as a single session to form a single time series for each individual, and two regressors of no interest were added to account for the session effect. A high-pass filter with a cutoff period of $256 \mathrm{~s}$ was applied. Group results were obtained using random-effects analyses by combining subject-specific summary statistics across the group as implemented in SPM2 (Penny and Holmes, 2003). The group results were then used for choosing the regions of interest (ROIs) for the effective connectivity analysis. To simplify the network, and because of the strong asymmetry in the activation clusters and well documented laterality of language processes, only lefthemisphere clusters larger than 50 voxels were included. This process resulted in four ROIs in the left hemisphere: FG, IFG, IPS, and LTC. Two regions were active in both tasks (i.e., FG and IFG), whereas the other two ROIs were each selectively active in one of the tasks (i.e., the IPS in the spelling task and the LTC in the rhyming task) (Fig. 1, Table 1).

Effective connectivity. Four regions of interest were specified in the left hemisphere for each individual: FG, IFG, IPS, and LTC. Regional responses were summarized as the principal eigenvariates of responses within a sphere centered on the most significant voxel for each subject. For the task-specific regions (LTC and IPS), this was a $6 \mathrm{~mm}$ sphere, to minimize the number of inactive voxels included. For the task-common regions (IFG and FG), we used a $10 \mathrm{~mm}$ sphere to include active voxels from both the spelling and rhyming tasks to enable the direct comparison of the effects of the two tasks in a single DCM model. Subject-specific maxima were defined operationally as the most significant voxels within $22 \mathrm{~mm}$ of the group maximum in the appropriate SPM. The SPMs for the task-specific regions (LTC and IPS) tested for rhyming and spelling, respectively. For the IFG and FG, the maxima across both tasks were averaged in each individual. Two subjects were excluded, because there were no significant clusters within $22 \mathrm{~mm}$ from the group-reference voxel (in IFG and LTC). Figure 2 (and Table 2, available at www.jneurosci.org as supplemental material) presents the location of the centers of the individual ROIs.

Effective connectivity analysis was performed using the DCM tool in SPM2 (Friston et al., 2003; Penny et al., 2004). In DCM, three sets of parameters are estimated: the direct influence of stimuli on regional activity, the intrinsic or latent connections between regions (i.e., the interregional influences in the absence of modulating experimental effects), and the changes in the intrinsic connectivity between regions induced by the experimental design (modulatory effects) (Mechelli et al., 
Table 1. Group mean activation for the spelling and rhyming tasks

\begin{tabular}{|c|c|c|c|c|c|c|c|}
\hline Task/region & $B A$ & Hemisphere & zscore & Voxels & $x$ & $y$ & $z$ \\
\hline \multicolumn{8}{|l|}{ Spelling } \\
\hline Inferior frontal gyrus & $45 / 46$ & L & 6.29 & 868 & -45 & 30 & 6 \\
\hline Fusiform/middle occipital gyrus & 37 & $\mathbf{L}$ & 5.43 & 399 & -42 & -60 & -18 \\
\hline Medial/ superior frontal gyri & $32 / 8$ & & 5.03 & 241 & -6 & 24 & 45 \\
\hline Inferior /middle frontal gyri & $45 / 8 / 9$ & $\mathrm{R}$ & 4.19 & 155 & 51 & 21 & 24 \\
\hline Superior/inferior parietal lobule/ precuneus & $19 / 40$ & L & 4.07 & 170 & -30 & -66 & 42 \\
\hline Superior parietal lobule / precuneus & $19 / 39$ & $\mathrm{R}$ & 3.94 & 60 & 30 & -63 & 42 \\
\hline \multicolumn{8}{|l|}{ Rhyming } \\
\hline Inferior/ middle frontal gyrus & $45 / 46 / 9$ & $\mathbf{L}$ & 5.2 & 733 & -45 & 30 & 18 \\
\hline Fusiform gyrus & $37 / 19$ & $\mathbf{L}$ & 5.2 & 342 & -45 & -60 & -21 \\
\hline Inferior frontal gyrus & 47 & $\mathrm{R}$ & 4.44 & 72 & 42 & 21 & -9 \\
\hline Middle temporal gyrus & 21 & $\mathbf{L}$ & 3.96 & 71 & -66 & -36 & -3 \\
\hline Superior frontal gyrus & 6 & & 3.94 & 52 & 3 & 15 & 57 \\
\hline Medial frontal gyrus & $9 / 8$ & & 3.9 & 85 & 6 & 36 & 45 \\
\hline
\end{tabular}

Left-hemisphere clusters appear in boldface. L, Left hemisphere; $R$, right hemisphere.

2003). Our analysis adopted a two-stage procedure that is formally identical to the summary statistic approach used in random effects analysis of neuroimaging data. However, in our case, the first level (subject-specific) models were DCMs, the parameters of which were the coupling and changes in coupling induced by task. The conditional expectations or modes of these parameters were taken to a second (between-subjects) level using the Bayesian random effects approach. The use of a Bayesian framework avoids the need for multiple comparisons adjustments when making inferences about the connection parameters (Friston et al., 2003). Subject-specific DCMs were fully and reciprocally connected with modulatory influences from both tasks specified on all the connections. Input stimuli (i.e., the rhyming and the spelling tasks) were modeled as exerting direct effects on the FG. A fully connected and fully modulated model was specified as a result of the scarcity of previous detailed anatomical knowledge of connectivity in humans, especially in the language system, and the highly reciprocal interconnectivity found in other systems in primate brains (Bullmore et al., 2000; Mechelli et al., 2002; Chaminade and Fonlupt, 2003). DCM enables this level of connectivity by using a Bayesian framework in model estimation (Friston et al., 2003). For each selectively active region, the modulatory effects were compared between tasks and between converging and diverging connections within task. Effects were considered significant if the posterior probability exceeded the threshold of $p>0.95$.

\section{Results}

The accuracy of performance was comparable in the spelling and rhyming tasks, with a mean accuracy of 98 and $97 \%$, respectively, in the experimental conditions and 97 and 98\%, respectively, in the control conditions. The reaction time was also comparable between the spelling and rhyming tasks with a mean reaction time of 868 and $916 \mathrm{~ms}$, respectively, in the experimental conditions and 769 and $763 \mathrm{~ms}$, respectively, in the control conditions. Reaction time was longer in the experimental conditions compared with the control condition in both tasks $\left[t_{(11)}=3.8 ; t_{(11)}=\right.$ $6.2(p<0.01)$; in the spelling and rhyming tasks, respectively].

\section{Conventional analysis}

Figure 1 and Table 1 present the patterns of activation in the spelling and in the rhyming tasks compared with baseline. The group maxima of left-hemisphere clusters (uncorrected $p<$ 0.001; extent threshold, 50 voxels) were used as reference for choosing the individual ROIs. For the spelling task, these ROIs included the inferior frontal gyrus (BA 45/46), the fusiform gyrus (BA 37), and the intraparietal sulcus, including the precuneus and parts of the superior and inferior parietal lobules (BA 19/40). In the rhyming task, these clusters included the middle/inferior

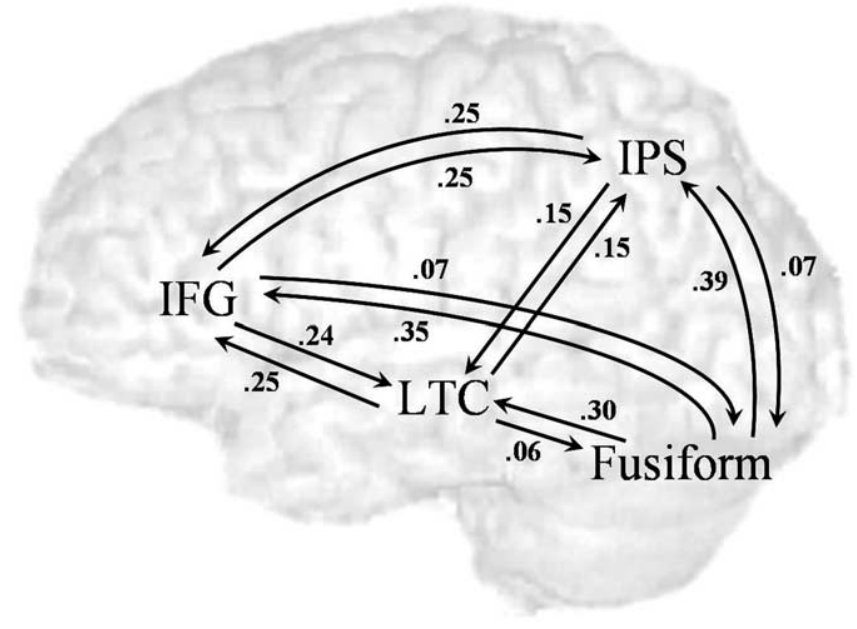

Figure 3. Intrinsic connections. The average strengths of influences across individuals are presented. Significant influences (posterior probability, $>0.95$ ) are presented.

frontal gyri (BA 46/45/9), the left fusiform gyrus (BA 37), and the superior and middle temporal gyri (BA 21/22).

\section{Effective connectivity analysis}

\section{Intrinsic connections}

Figure 3 shows that all intrinsic influences among regions (i.e., connections that are independent of the task) were significant. The influence of FG on all other regions had posterior probabilities of 1.0. The influence of IFG on FG, IPS, and LTC had posterior probabilities of $0.99,1.0$, and 1.0 , respectively. The influence of IPS on FG, IFG, and LTC had posterior probabilities of 0.985 , 1.0, and 0.999, respectively, and the influence of LTC on FG, IFG, and IPS had posterior probabilities of $0.967,1.0$, and 0.994 , respectively.

\section{Effect of the spelling task}

The direct effect of the spelling task on the FG was weak (0.04) but significant $(p=1.0)$. Figure $4 A$ shows the modulatory effects of the spelling task. The spelling task showed significant modulatory effects on all converging influences into IPS ( $p=1.0$ for the effects of FG, IFG, and LTC on IPS). In addition, the spelling task significantly modulated all converging influences into IFG ( $p=$ 0.996, 0.998, and 0.999 for FG, IPS, and LTC). The effect of the direction of influence (converging vs diverging influence) was tested for the modulatory effects of the spelling task on couplings 


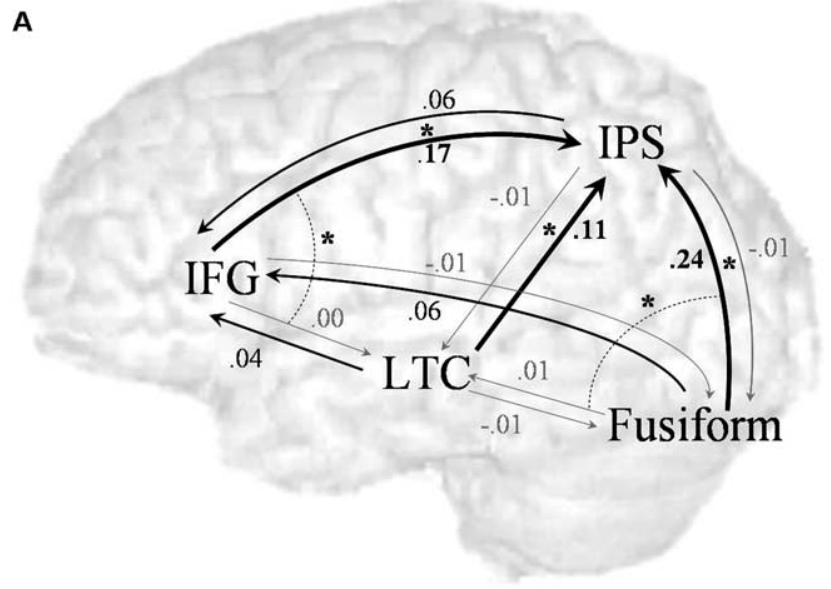

B

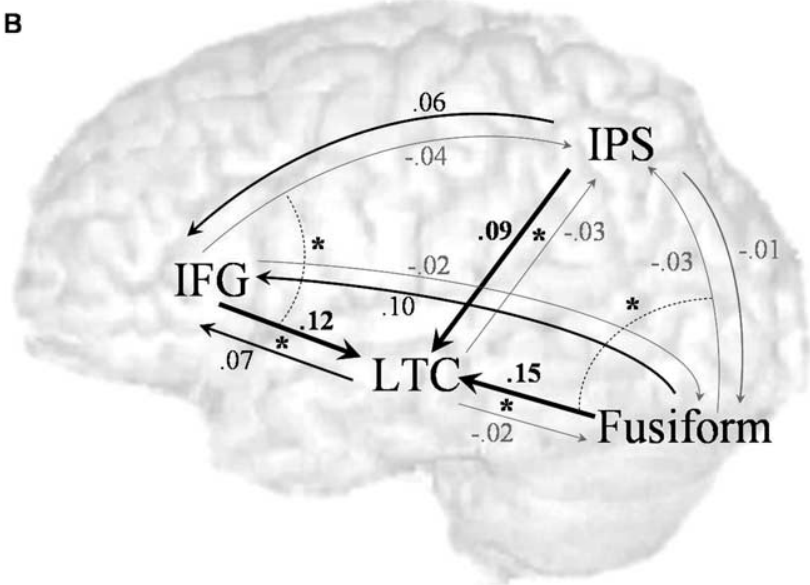

Figure 4. Modulatory effects of the spelling $(\boldsymbol{A})$ and rhyming $(\boldsymbol{B})$ tasks. The average strengths of effects across individuals are presented. Significant effects (posterior probability, $>0.95$ ) are presented in black and nonsignificant effect in gray. Pairs of effects that are significantly different are indicated by * (and connected by a dotted arc in distant pairs) with the stronger effect indicated in bold. For example, in $A$, the dotted arc on the right indicates that the fusiform area had a significantly greater influence on the IPS than on the LTC in the spelling task.

involving the IPS. The effect of direction was tested in three separate contrasts, corresponding to the three regions coupled with IPS. All the converging influences of other regions on IPS were modulated significantly more than the diverging influences of IPS on other regions ( $p=1.0,0.999$, and 1.0 for FG, IFG, and LTC). A contrast of the interaction between selectively active region (IPS vs LTC) and the direction of influence (converging vs diverging) was tested for the modulatory effect of the spelling task on the coupling with IFG and FG. The analyses showed significant interactions between selectively active region and direction of influence for the IFG ( $p=0.997)$ and FG $(p=0.998)$. The direct comparison between selectively active regions showed that the converging influence of FG and IFG on IPS were modulated by the spelling task significantly more than the converging influence of these regions on LTC ( $p=0.998$ for FG and $p=0.996$ for the IFG) (Fig. 4A, dotted arcs). However, the diverging influences from IPS and LTC (to FG and IFG) were not differentially modulated by the spelling task ( $p=0.688$ for FG and $p=0.909$ for the IFG).

\section{Effect of the rhyming task}

The direct effect of the rhyming task on the FG was weak (0.03) but significant ( $p=1.0$ ). Figure $4 B$ shows the modulatory effects of the rhyming task. The rhyming task showed significant mod-

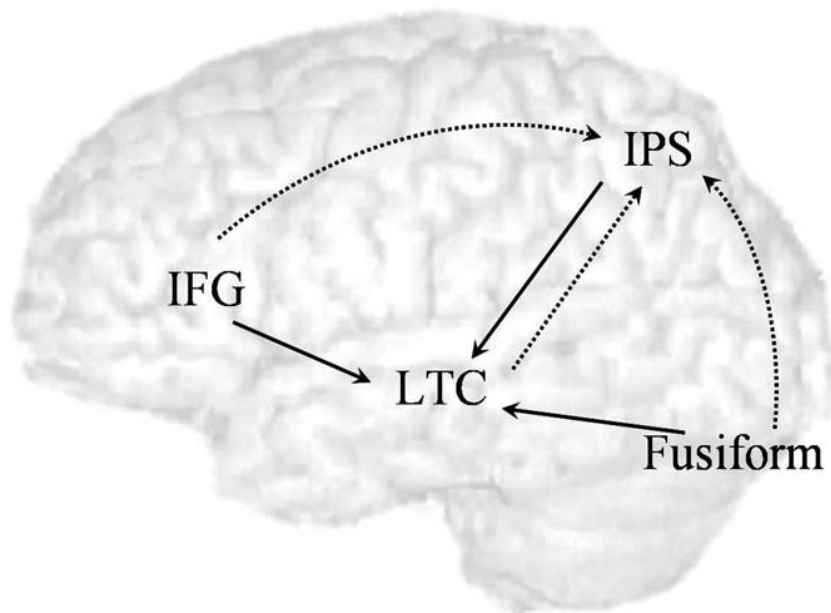

Figure 5. Differential modulatory effects between tasks. The dotted arrow indicates influences that are modulated by the spelling task significantly more than by the rhyming task. The solid arrow indicates influences that are modulated by the rhyming task significantly more than by the spelling task.

ulatory effects on all converging influences into LTC ( $p=1.0$ for the effects of FG, IFG, and IPS on LTC). In addition, the rhyming task significantly modulated all converging influences into IFG ( $p=1.0,0.993,0.998)$ for the effects of FG, IPS, and LTC on IFG). We repeated the identical analysis done on the spelling task for the effect of the rhyming task. The effect of the direction of influence (converging vs diverging influence) was tested for the modulatory effects of the rhyming task on couplings involving the LTC. The effect of direction was tested in three separate contrasts, corresponding to the three regions coupled with LTC. All the converging influences of other regions on LTC were modulated significantly more than the diverging influences of LTC on other regions ( $p=1.0,0.967$, and 1.0 for FG, IFG, and IPS). A contrast of the interaction between selectively active region (IPS vs LTC) and the direction of influence (converging vs diverging) was tested for the modulatory effect of the rhyming task on the coupling with IFG and FG. The analyses showed significant interactions between selectively active region and direction of influence for the IFG ( $p=0.999)$ and FG ( $p=0.999)$. The direct comparison between selectively active regions showed that the converging influence of IFG and FG on LTC were modulated by the rhyming task significantly more than the converging influence of these regions on IPS ( $p=0.999$ for FG and $p=1.0$ for the IFG) (Fig. 4B, dotted arcs). However, the diverging influences from IPS and LTC (to IFG and FG) were not differentially modulated by the rhyming task ( $p=0.891$ for FG and $p=0.846$ for the IFG).

\section{Between-task comparisons}

Figure 5 shows modulatory effects that were significantly different between tasks. A contrast testing the interaction of task (spelling vs rhyming) and direction of influence (converging vs diverging) was tested for each pair of regions that involved the selectively active regions. The interaction of task and direction of influence was significant in all pairs: FG-IPS ( $p=0.999), \mathrm{FG}-$ LTC $(p=0.990), \operatorname{IFG-IPS~}(p=1.0), \operatorname{IFG-LTC~}(p=0.959)$, and LTC-IPS $(p=1.0)$. Direct comparisons between directions showed that converging influences of all regions on IPS were modulated by the spelling task significantly more than by the rhyming task $(p=1.0,0.999$, and 1.0 for the effects of the FG, 
IFG, and LTC on IPS, respectively). However, the diverging influences of the IPS on FG and IFG were not differentially modulated by the tasks ( $p=0.503$ and 0.551 , respectively). In a similar way, direct comparisons between directions showed that converging influences of all regions on LTC were modulated by the rhyming task significantly more than by the spelling task ( $p=$ 0.993, 0.986, and 0.996 for the effects of FG, IFG, and IPS on LTC, respectively). However, the diverging influences of LTC on FG and IFG were not differentially modulated by the tasks ( $p=$ 0.698 and 0.865 , respectively).

\section{Discussion}

We used two lexical tasks using written words, one requiring the processing of phonological information (rhyming), the other orthographic information (spelling). Activations specific to rhyming and spelling were identified in comparison to a baseline task requiring the visual processing of nonverbal symbols. The lefthemisphere network we identified contained four components. Two of these, the IFG (partially overlapping Broca's area) and the FG (visual association cortex), were activated by both tasks, whereas LTC and IPS displayed task-specific activations for phonology and orthography, respectively (Fig. 1).

The selective activation in the LTC for the rhyming task and in the IPS for the spelling task is in accord with previous studies showing the involvement of these regions in phonological and visuospatial processing, respectively. The LTC region was involved in rhyming judgments performed on visually presented letters (Paulesu et al., 1996; Pugh et al., 1996), nonwords (Paulesu et al., 1996; Xu et al., 2001), and real words (Crosson et al., 1999; Kareken et al., 2000; Lurito et al., 2000; Xu et al., 2001). Additional studies had also suggested that activation in the superior temporal gyrus region of the LTC may reflect access to phonologic word forms (Howard et al., 1992; Binder et al., 1994; Giraud and Price, 2001). Many experiments have reported IPS activations in spatial imagery (Cohen et al., 1996; Alivisatos and Petrides, 1997; Kosslyn et al., 1998), as well as in spatial and verbal working memory tasks (Paulesu et al., 1993; Owen et al., 1996; Salmon et al., 1996; LaBar et al., 1999; Cabeza and Nyberg, 2000; Homae et al., 2003). Thus, activation of IPS in our spelling task could reflect the access and maintenance of visuospatial word representations necessary for orthographic judgments.

The analysis of task-independent (i.e., intrinsic) connectivity showed that our network was tightly and reciprocally interconnected. The intrinsic connectivity depicted in Figure 3 shows that each of the four components can potentially modulate activity in any of the others and can potentially be modulated by any of the others. The presence of full interconnectivity is consistent with neuroanatomical experiments that have shown that the analogous parts of the monkey brain are reciprocally interconnected through monosynaptic pathways (Jacobson and Trojanowski, 1977; Mesulam et al., 1977; Pandya and Yeterian, 1985).

The most striking outcome of the DCM analysis was that each verbal-processing task led to a strengthening of neural influences converging on the site specialized for that task, LTC for rhyming, and IPS for spelling (Figs. 4A, $B, 5$ ). It appears, therefore, that the cognitive goal is achieved through the integration of information in specialized areas more so than through the top-down control such areas exert on other parts of the network. The role of the IPS and LTC as convergence zones is consistent with the suggestion that these transmodal association areas are sites enabling the multidimensional synthesis of knowledge (Mesulam, 1998). Damasio (1989) suggests that the binding of different aspects of the stimuli in convergence zones does not occur only in high- level prefrontal cortices but in multiple levels of neural representations in multiple cortical regions. The integration of taskspecific information in the IPS and LTC in the current study is consistent with the notion that multiple convergence zones are distributed in posterior brain regions.

A comparison of either verbal task to the baseline nonverbal task revealed a polarity of IFG-FG interactions that was significantly stronger in the sensory-fugal direction (i.e., from FG to IFG) (Fig. $4 A, B$ ). This finding is in accord with the role of the FG as the major relay of visual stimuli into the network, because it encodes a broad range of complex visual percepts, including verbal and nonverbal stimuli (Kanwisher et al., 1997; Cohen et al., 2000; Haxby et al., 2001). In contrast, the IFG, in its capacity as a major component of the language network, is more responsive to verbal than nonverbal information emanating from FG (Price et al., 1994; Binder et al., 1996; Price, 2000). Neither task led to a strengthening of top-down influences on the FG, suggesting that the performance of the verbal task does not depend on a modification of visual encoding at the unimodal stage of processing. This is in keeping with many other examples in which the transformations from sensation to cognition occur within transmodal rather than unimodal areas, so that the fidelity of modalityspecific encoding remains protected. This encoding scheme would preserve sensory fidelity and allow the same information to be used by other cognitive networks (Mesulam, 1998).

Our results also show that the IFG and FG may dynamically switch their functional affiliation with IPS or LTC, depending on the requirements of the task. The activation of different parts of the brain for identical inputs, depending on the prevailing cognitive goal, is consistent with several previous reports (Seeck et al., 1995; Stephan et al., 2003). For example, Seeck et al. (1995), using depth electrode recordings, showed that identical faces evoked responses in inferotemporal cortex when the goal was perceptual processing, in the amygdala when the goal was the recognition of familiarity, and in prefrontal cortex when the goal was to maintain the information in working memory. The question may be asked how such task-specific shifts of sensitivity to sensory stimuli are achieved. The current DCM analysis suggests a plausible scenario according to which the IFG is a nodal point wherein the activation of other network components is coordinated during language tasks. According to this scenario, the initiating event would be the activation of the IFG by verbal stimuli. Depending on task requirements, the IFG would then generate neural signals that differentially sensitize LTC versus IPS to the input from FG. The sensitized area would show greater resonance with neural activity in the FG, leading to a strengthening of its afferent connectivity with FG, as shown in Figures 4 and 5. The verbal stimuli would then be decoded in terms of their phonological or orthographic information, depending on the location of the preferentially sensitized network component. In this sense, the IFG would play a key role in biasing the network toward task-specific requirements (Milham et al., 2003; Perianez et al., 2004). The DCM analysis does not definitively establish the existence of this scenario, but it provides evidence that makes it plausible and open to empirical verification by methods that have better temporal resolution.

The conjectured role of the IFG in the modulation of posterior brain regions for maintaining the cognitive set for orthographic and phonological processing is consistent to some extent with the suggested role of the IFG in the control of verbal memory and semantic processing. Previous studies have shown that the left IFG is involved in semantic tasks that require effortful retrieval of semantic representation (Fiez, 1997), semantic elaboration dur- 
ing the encoding phase of a verbal memory task (Buckner et al., 1999), or selection among competing semantic representations (Thompson-Schill et al., 1999). It has been suggested that the IFG acts as a semantic executive system, which controls the access, retrieval, selection, and gating of semantic information by the modulation or reactivation of representations in posterior brain regions (Wagner et al., 1997; Poldrack et al., 1999; Roskies et al., 2001). However, in contrast to the notion that the IFG serves as a final site for synthesis of task-relevant attributes of the representations (Poldrack et al., 1999), we suggest that the integration of task-specific information is performed by the posterior regions.

Together, our results suggests that in rhyming and spelling tasks of visually presented words, the regions that are selectively active in each task serve as regions of integration of information relevant to the task. The main role of these selectively active regions is in integrating information relevant to the specific task to make the orthographic or phonological judgment. Our findings suggest that integration of information may be distributed in posterior cortical regions corresponding to the specific task, rather than being restricted to the prefrontal cortex. Furthermore, the role of the task-selective areas is to provide a differential synthesis of incoming information rather than providing differential control signals influencing the activity of other network components. Our results also suggest that task-dependent shifts in effective connectivity are likely to be mediated through topdown modulations from the IFG to the task-selective regions in a way that differentially enhances their sensitivity to incoming information.

\section{References}

Alivisatos B, Petrides M (1997) Functional activation of the human brain during mental rotation. Neuropsychologia 35:111-118.

Binder JR, Rao SM, Hammeke TA, Yetkin FZ, Jesmanowicz A, Bandettini PA, Wong EC, Estkowski LD, Goldstein MD, Haughton VM, Hyde JS (1994) Functional magnetic resonance imaging of human auditory cortex. Ann Neurol 35:662-672.

Binder JR, Frost JA, Hammeke TA, Rao SM, Cox RW (1996) Function of the left planum temporale in auditory and linguistic processing. Brain 119:1239-1247.

Booth JR, Burman DD, Meyer JR, Gitelman DR, Parrish TB, Mesulam MM (2002) Functional anatomy of intra- and cross-modal lexical tasks. NeuroImage 16:7-22.

Bowey JA (1990) Orthographic onsets and rimes as functional units of reading. Mem Cognit 18:419-427.

Buckner RL, Kelley WM, Petersen SE (1999) Frontal cortex contributes to human memory formation. Nat Neurosci 2:311-314.

Bullmore E, Horwitz B, Honey G, Brammer M, Williams S, Sharma T (2000) How good is good enough in path analysis of fMRI data? NeuroImage 11:289-301.

Cabeza R, Nyberg L (2000) Imaging cognition II: an empirical review of 275 PET and fMRI studies. J Cogn Neurosci 12:1-47.

Chaminade T, Fonlupt P (2003) Changes of effective connectivity between the lateral and medial parts of the prefrontal cortex during a visual task. Eur J Neurosci 18:675-679.

Cohen L, Dehaene S, Naccache L, Lehericy S, Dehaene-Lambertz G, Henaff MA, Michel F (2000) The visual word form area: spatial and temporal characterization of an initial stage of reading in normal subjects and posterior split-brain patients. Brain 123:291-307.

Cohen MS, Kosslyn SM, Breiter HC, DiGirolamo GJ, Thompson WL, Anderson AK, Bookheimer SY, Rosen BR, Belliveau JW (1996) Changes in cortical activity during mental rotation-a mapping study using functional MRI. Brain 119:89-100.

Crosson B, Rao SM, Woodley SJ, Rosen AC, Bobholz JA, Mayer A, Cunningham JM, Hammeke TA, Fuller SA, Binder JR, Cox RW, Stein EA (1999) Mapping of semantic, phonological, and orthographic verbal working memory in normal adults with functional magnetic resonance imaging. Neuropsychology 13:171-187.
Damasio AR (1989) The brain binds entities and events by multiregional activation from convergence zones. Neural Comput 1:123-132.

Fiez JA (1997) Phonology, semantics, and the role of the left inferior prefrontal cortex. Hum Brain Mapp 5:79-83.

Friston KJ, Harrison L, Penny W (2003) Dynamic causal modelling. NeuroImage 19:1273-1302.

Giraud AL, Price CJ (2001) The constraints functional neuroimaging places on classical models of auditory word processing. J Cogn Neurosci 13:754-765.

Haxby JV, Gobbini MI, Furey ML, Ishai A, Schouten JL, Pietrini P (2001) Distributed and overlapping representations of faces and objects in ventral temporal cortex. Science 293:2425-2430.

Henson R, Buchel C, Josephs O, Friston K (1999) The slice-timing problem in event-related fMRI. NeuroImage 9:S125.

Homae F, Yahata N, Sakai KL (2003) Selective enhancement of functional connectivity in the left prefrontal cortex during sentence processing. NeuroImage 20:578-586.

Horwitz B, Rumsey JM, Donohue BC (1998) Functional connectivity of the angular gyrus in normal reading and dyslexia. Proc Natl Acad Sci USA 95:8939-8944.

Howard D, Patterson K, Wise R, Brown WD, Friston K, Weiller C, Frackowiak R (1992) The cortical localization of the lexicons. Positron emission tomography evidence. Brain 115:1769-1782.

Jacobson S, Trojanowski JQ (1977) Prefrontal granular cortex of the rhesus monkey. I. Intrahemispheric cortical afferents. Brain Res 132:209-233.

Kanwisher N, McDermott J, Chun MM (1997) The fusiform face area: a module in human extrastriate cortex specialized for face perception. J Neurosci 17:4302-4311.

Kareken DA, Lowe M, Chen SHA, Lurito J, Mathews V (2000) Word rhyming as a probe of hemispheric language dominance with functional magnetic resonance imaging. Neuropsychiatry Neuropsychol Behav Neurol 13:264-270.

Kosslyn SM, Digirolamo GJ, Thompson WL, Alpert NM (1998) Mental rotation of objects versus hands: neural mechanisms revealed by positron emission tomography. Psychophysiology 35:151-161.

LaBar KS, Gitelman DR, Parrish TB, Mesulam M (1999) Neuroanatomic overlap of working memory and spatial attention networks: a functional MRI comparison within subjects. NeuroImage 10:695-704.

Lurito JT, Kareken DA, Lowe MJ, Chen SH, Mathews VP (2000) Comparison of rhyming and word generation with FMRI. Hum Brain Mapp 10:99-106.

McIntosh AR (2000) Towards a network theory of cognition. Neural Netw 13:861-870.

McIntosh AR, Grady CL, Ungerleider LG, Haxby JV, Rapoport SI, Horwitz B (1994) Network analysis of cortical visual pathways mapped with PET. J Neurosci 14:655-666.

Mechelli A, Penny WD, Price CJ, Gitelman DR, Friston KJ (2002) Effective connectivity and intersubject variability: using a multisubject network to test differences and commonalities. NeuroImage 17:1459-1469.

Mechelli A, Price CJ, Noppeney U, Friston KJ (2003) A dynamic causal modeling study on category effects: bottom-up or top-down mediation? J Cogn Neurosci 15:925-934.

Mesulam MM (1981) A cortical network for directed attention and unilateral neglect. Ann Neurol 10:309-325.

Mesulam MM (1998) From sensation to cognition. Brain 121:1013-1052.

Mesulam MM, Van Hoesen GW, Pandya DN, Geschwind N (1977) Limbic and sensory connections of the inferior parietal lobule (area PG) in the rhesus monkey: a study with a new method for horseradish peroxidase histochemistry. Brain Res 136:393-414.

Milham MP, Banich MT, Barad V (2003) Competition for priority in processing increases prefrontal cortex's involvement in top-down control: an event-related fMRI study of the Stroop task. Brain Res Cogn Brain Res 17:212-222.

Owen AM, Evans AC, Petrides M (1996) Evidence for a two-stage model of spatial working memory processing within the lateral frontal cortex: a positron emission tomography study. Cereb Cortex 6:31-38.

Pandya DN, Yeterian EH (1985) Architecture and connections of cortical association areas. Cereb Cortex 4:3-61.

Paulesu E, Frith CD, Frackowiak RS (1993) The neural correlates of the verbal component of working memory. Nature 362:342-345.

Paulesu E, Frith U, Snowling M, Gallagher A, Morton J, Frackowiak RSJ, Frith 
CD (1996) Is developmental dyslexia a disconnection syndrome? Evidence from PET scanning. Brain 119:143-157.

Penny WD, Holmes A (2003) Random effects analysis. In: Human brain function, Ed 2 (Frackowiak RSJ, Friston KJ, Frith CD, eds), pp 843-850. San Diego: Academic.

Penny WD, Stephan KE, Mechelli A, Friston KJ (2004) Comparing dynamic causal models. NeuroImage 22:1157-1172.

Perianez JA, Maestu F, Barcelo F, Fernandez A, Amo C, Ortiz Alonso T (2004) Spatiotemporal brain dynamics during preparatory set shifting: MEG evidence. NeuroImage 21:687-695.

Poldrack RA, Wagner AD, Prull MW, Desmond JE, Glover GH, Gabrieli JDE (1999) Functional specialization for semantic and phonological processing in the left inferior prefrontal cortex. NeuroImage 10:15-35.

Price CJ (2000) The anatomy of language: contributions from functional neuroimaging. J Anat 197:335-359.

Price CJ, Wise RJ, Watson JD, Patterson K, Howard D, Frackowiak RS (1994) Brain activity during reading. The effects of exposure duration and task. Brain 117:1255-1269.

Pugh KR, Shaywitz BA, Shaywitz SE, Constable RT, Skudlarski P, Fulbright RK, Bronen RA, Shankweiler DP, Katz L, Fletcher JM, Gore JC (1996) Cerebral organization of component processes in reading. Brain 119:1221-1238.

Pugh KR, Mencl WE, Shaywitz BA, Shaywitz SE, Fulbright RK, Constable RT, Skudlarski P, Marchione KE, Jenner AR, Fletcher JM, Liberman AM, Shankweiler DP, Katz L, Lacadie C, Gore JC (2000) The angular gyrus in developmental dyslexia: task-specific differences in functional connectivity within posterior cortex. Psychol Sci 11:51-56.

Roskies AL, Fiez JA, Balota DA, Raichle ME, Petersen SE (2001) Taskdependent modulation of regions in the left inferior frontal cortex during semantic processing. J Cogn Neurosci 13:829-843.

Salmon E, VanderLinden M, Collette F, Delfiore G, Maquet P, Degueldre C, Luxen A, Franck G (1996) Regional brain activity during working memory tasks. Brain 119:1617-1625.

Seeck M, Schomer D, Mainwaring N, Ives J, Dubuisson D, Blume H, Cosgrove R, Ransil BJ, Mesulam MM (1995) Selectively distributed processing of visual object recognition in the temporal and frontal lobes of the human brain. Ann Neurol 37:538-545.

Stephan KE, Marshall JC, Friston KJ, Rowe JB, Ritzl A, Zilles K, Fink GR (2003) Lateralized cognitive processes and lateralized task control in the human brain. Science 301:384-386.

Thompson-Schill SL, D’Esposito M, Kan IP (1999) Effects of repetition and competition on activity in left prefrontal cortex during word generation. Neuron 23:513-522.

Wagner AD, Desmond JE, Demb JB, Glover GH, Gabrieli JED (1997) Semantic repetition priming for verbal and pictoral knowledge: a functional MRI study of left inferior prefrontal cortex. J Cogn Neurosci 9:714-726.

Xu B, Grafman J, Gaillard WD, Ishii K, Vega-Bermudez F, Pietrini P, ReevesTyer P, DiCamillo P, Theodore W (2001) Conjoint and extended neural networks for the computation of speech codes: the neural basis of selective impairment in reading words and pseudowords. Cereb Cortex 11:267277. 